\title{
Energy frontier DIS at CERN: the LHeC and the FCC- eh, PERLE
}

\author{
G. Arduini ${ }^{1}$, O.Brüning \\ CERN \\ 1211 Geneva 23, Switzerland \\ E-mail: gianluigi.arduiniecern.ch
}

\section{Klein}

Department of Physics, University of Liverpool

The Oliver Lodge Laboratory, Liverpool L69 7ZE, United Kingdom

E-mail: Max.Kleineliverpool.ac.uk

\section{for the LHeC and FCC-eh Study Groups and the PERLE Collaboration}

\begin{abstract}
Energy-frontier Deep Inelastic Scattering (DIS) can be realised at CERN through an energy recovery linac (ERL) that would produce $60 \mathrm{GeV}$ electrons to collide with the High Luminosity (HL)-Large Hadron Collider (LHC) or, eventually, with the High Energy (HE)-LHC or the Future Circular Collider (FCC) hadron beams. It would deliver lepton-proton (nucleus) collisions with centre of mass energies in the range $0.8-3.5 \mathrm{TeV}$ per nucleon, and luminosities exceeding $10^{34}$ $\left(5 \times 10^{32}\right) \mathrm{cm}^{-2} \mathrm{~s}^{-1}$ in electron-proton (electron-lead ion) beam operation. Such machine would provide a huge physics program, with the highest resolution microscope for hadron structure, rich Higgs, top and precision Electro-Weak (EW) physics, large possibilities for Beyond Standard Model (BSM) searches, and a unique top-energy nuclear physics facility with eventual access to a new regime of QCD at high partonic densities. All these aspects have strong complementarities with the respective, concurrent pp and AA programs. In this paper we review the $\mathrm{LHeC}, \mathrm{HE}-\mathrm{LHeC}$ and FCC-eh proposals at CERN, with emphasis on the accelerator and infrastructure aspects. We also review the project of an ERL demonstrator, PERLE, under consideration to be built at LAL Orsay.
\end{abstract}

XXVI International Workshop on Deep-Inelastic Scattering and Related Subjects (DIS2018) 16-20 April 2018

Kobe, Japan

\footnotetext{
${ }^{1}$ Speaker 


\section{Introduction}

Initiated by the CERN Scientific Policy Committee (SPC) in 2007 and supported by CERN, the European Committee for Future Accelerators (ECFA) and the Nuclear Physics European Collaboration Committee (NuPECC) a series of studies have been performed to determine the parameters and feasibility of an electron hadron collider at CERN as a possible upgrade of the LHC complementary to its High Luminosity upgrade. This would provide CERN and the Particle Physics community with the cleanest high resolution microscope to study the structure of protons, neutrons and nuclei with unprecedented precision and would further extend the range of searches for Physics Beyond the Standard Model.

More recently the studies have been extended to explore the technical solutions and the potential of electron-hadron collision at even higher centre-of-mass energies that could be made available with Future Circular Colliders.

\section{LHeC at CERN}

The design of an electron-proton (ion) collider (Large electron Hadron Collider - LHeC), in which a newly built accelerator provides $60 \mathrm{GeV}$ electron beams colliding with one of the hadron beams in the LHC, has been conducted at CERN and the results published in 2012 [1]. The main assumptions and constraints considered in the study were:

- electron-hadron operation in parallel with high luminosity hadron-hadron collisions in LHC/HL-LHC;

- centre-of-mass collision energy in the TeV scale;

- power consumption of the electron accelerator smaller than $100 \mathrm{MW}$;

- peak luminosity approaching $10^{34} \mathrm{~cm}^{-2} \mathrm{~s}^{-1}$;

- integrated luminosity exceeding by at least two orders of magnitude that achieved by HERA at DESY.

Two possible options have been studied and presented in the LHeC Conceptual Design Report (CDR) (see Fig. 1):

- Ring-Ring option including a new ring in the LHC tunnel with bypasses around existing experiments and a new $10 \mathrm{GeV}$ injector;

- Linac-Ring option based on a recirculating linac with energy recovery.

The Linac-Ring options with Energy Recovery (Energy Recovery Linac - ERL) satisfies the above assumptions and constraints and provides an elegant and (energy and cost) efficient solution that is compatible with the HL-LHC installation and operation scenarios. For that reason it has been selected as the baseline design for LHeC. It consists of two $1 \mathrm{~km}$ long, $10 \mathrm{GeV}$ superconducting (SC) linacs operated in $\mathrm{CW}$ connected by three pairs of arcs to allow three accelerating and three decelerating passes in the linacs for a total circumference corresponding to on third of the LHC circumference (see Fig. 2). The length of the high energy return arc following the interaction point should be such to provide a $1 / 2 \mathrm{RF}$ period wavelength shift to allow the deceleration of the beam in the linac structures in three passes down to the injection energy and its safe disposal. SC Cavities with an unloaded quality factor $\mathrm{Q}_{0}$ exceeding $10^{10}$ are required to minimize the requirements on the cryogenic cooling power and to allow an efficient ERL operation. The choice of having three accelerating and three decelerating passes implies that the 
circulating current in the linacs is six times the current colliding at the Interaction Point (IP) with the hadron beam.

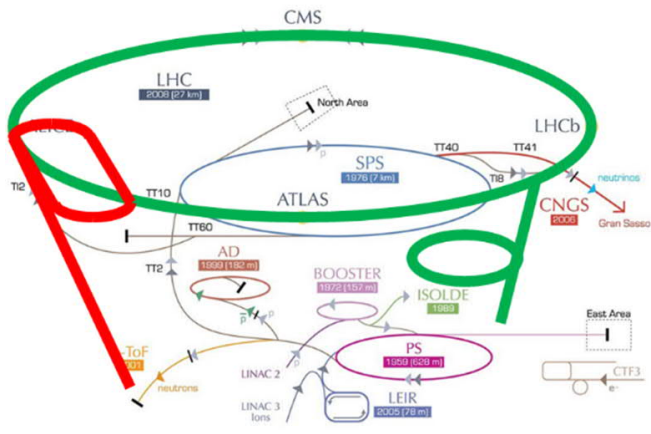

Fig. 1: Schematic layout of the Linac-Ring (red) and Ring-Ring (green) options for $\mathrm{LHeC}$.

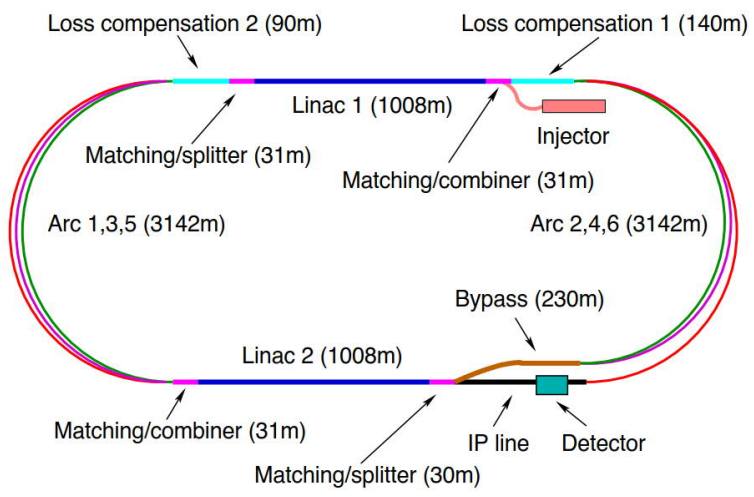

Fig. 2: Schematic layout of the $\mathrm{LHeC}$ design based on an Energy Recovery Linac.

The choice of an Energy Recovery Linac combines the advantages of a high brightness beam, essentially determined by the brightness at the source output, that can be matched in size to that of the HL-LHC proton beam, with storage ring beam powers while keeping the installed RF power at acceptable levels thanks to the energy recovery principle that allows disposing the beam at lower energy with correspondingly reduced power consumption and prompt and remnant radiation and avoiding performance limitations due to the beam-beam effect seen by the electron beam, which is a major performance limitation in many circular lepton colliders (e.g. LEP) and for the LHeC Ring-Ring option.

The main design parameters for LHeC based on the ERL concept are summarized in Table 1 both for ultimate LHC and the HL-LHC proton beam parameters [2] corresponding to a centreof-mass collision energy of $1.3 \mathrm{TeV}$.

\begin{tabular}{|l|c|c|c|c|}
\hline \multirow{2}{*}{} & \multicolumn{2}{|l|}{ CDR - LHeC } & \multicolumn{2}{l|}{ HL-LHeC } \\
\cline { 2 - 5 } & $\mathrm{p}$ & $\mathrm{e}^{-}$ & $\mathrm{p}$ & $\mathrm{e}^{-}$ \\
\hline Beam Energy $[\mathrm{GeV}]$ & 7000 & 60 & 7000 & 60 \\
\hline Luminosity $\left[10^{33} \mathrm{~cm}^{-2} \mathrm{~s}^{-1}\right]$ & \multicolumn{2}{|c|}{1} & \multicolumn{2}{c|}{8} \\
\hline Normalized emittance $\gamma \varepsilon_{x, y}[\mu \mathrm{m}]$ & 3.75 & 50 & 2.5 & 20 \\
\hline Beta function $\beta_{x, y}^{*}[\mathrm{~m}]$ & 0.1 & 0.12 & 0.05 & 0.1 \\
\hline R.M.S. beam size $\sigma_{x, y}^{*}[\mu \mathrm{m}]$ & 7 & 7 & 4 & 4 \\
\hline Beam Current @ IP [mA] & 860 & 6.4 & 1100 & 15 \\
\hline Bunch Spacing @ IP [ns] & 25 & 25 & 25 & 25 \\
\hline Bunch Population $\left[10^{11}\right]$ & 1.7 & 0.01 & 2.2 & 0.023 \\
\hline Bunch charge [nC] & 27 & 0.16 & 35 & 0.37 \\
\hline
\end{tabular}

Table 1. LHeC main design parameters.

The consideration of the HL-LHC proton beam [3] parameters together with a small beta function at the interaction point and an increased electron current (achieved and exceeded with available DC electron guns) allows approaching peak luminosities of $10^{34} \mathrm{~cm}^{-2} \mathrm{~s}^{-1}$ and integrated luminosities of approximately $1 \mathrm{ab}^{-1}$ in the HL-LHC lifetime, and opens the way to the study of the Higgs boson properties in electron proton collisions [4]. However this requires the demonstration of operating a high energy ERL with the very high current of $90 \mathrm{~mA}$ inside the 
SRF structures and corresponding to a virtual beam power (product of the beam current at the IP times the maximum beam energy) of almost $1 \mathrm{GW}(0.9 \mathrm{GW})$ while keeping the energy consumption for the ERL operation below 100MW!

\section{HE-LHeC and FCC-eh}

A further increase in the electron-proton centre-of-mass collision energy could be achieved with the proton beams of the High-Energy LHC (HE-LHC) [2,5], a collider that could be installed in the existing $26.7 \mathrm{~km}$ LHC tunnel deploying high field dipole magnets, as being designed in the frame of the FCC study and based on $16 \mathrm{~T}$ FCC dipole magnets, or the $50 \mathrm{TeV}$ proton beam of the Future Circular Collider (FCC-hh) [2,6,7] in a $97.7 \mathrm{~km}$ new tunnel. This novel FCC-eh electron-proton collider would enable DIS physics at $\sqrt{\mathrm{s}}=3.5 \mathrm{TeV}$ with a luminosity of the order of $10^{34} \mathrm{~cm}^{-2} \mathrm{~s}^{-1}$ in synchronous ep and pp operation. The physics programme reaches values as small as $10^{-7}$ of Bjorken $\mathrm{x}$ in DIS scattering and enables clean Higgs physics in addition to offer a unique discovery potential in QCD and beyond the Standard Model.

The main parameters of the HE-LHeC and FCC-eh colliders are summarized in Table 2 [2].

\begin{tabular}{|l|c|c|c|c|}
\hline \multirow{2}{*}{} & \multicolumn{2}{|c|}{ HE-LHeC $\sqrt{\mathrm{s}=1.7 \mathrm{TeV}}$} & \multicolumn{2}{c|}{ FCC-eh $\sqrt{\mathrm{s}}=3.5 \mathrm{TeV}$} \\
\cline { 2 - 5 } & $\mathrm{p}$ & $\mathrm{e}^{-}$ & $\mathrm{p}$ & $\mathrm{e}^{-}$ \\
\hline Beam Energy $[\mathrm{GeV}]$ & 12500 & 60 & 50000 & 60 \\
\hline Luminosity $\left[10^{33} \mathrm{~cm}^{-2} \mathrm{~s}^{-1}\right]$ & \multicolumn{2}{|c|}{12} & \multicolumn{2}{c|}{15} \\
\hline Normalized emittance $\gamma \varepsilon_{\mathrm{x}, \mathrm{y}}[\mu \mathrm{m}]$ & 2.5 & 10 & 2.2 & 10 \\
\hline Beta function $\beta_{\mathrm{x}, \mathrm{y}}^{*}[\mathrm{~m}]$ & 0.1 & 0.2 & 0.15 & 0.05 \\
\hline R.M.S. beam size $\sigma_{\mathrm{x}, \mathrm{y}}^{*}[\mu \mathrm{m}]$ & 4 & 4 & 2.5 & 2 \\
\hline Beam Current @ IP [mA] & 1250 & 20 & 500 & 20 \\
\hline Bunch Spacing @ IP [ns] & 25 & 25 & 25 & 25 \\
\hline Bunch Population $\left[10^{11}\right]$ & 2.5 & 0.03 & 1 & 0.03 \\
\hline Bunch charge [nC] & 40 & 0.5 & 16 & 0.5 \\
\hline
\end{tabular}

Table 2. HE-LHeC and FCC-eh main design parameters.

The corresponding parameters for electron-lead ion operation for LHeC, HE-LHeC and FCC-eh can be found in [2].

\section{Design concepts/choices and challenges}

In this section the main challenges underlying the design concepts and choices for the LHeC, HE-LHeC and FCC-eh colliders will be briefly outlined, in particular those related to the Interaction Region (IR) layout and magnet designs and to the ERL RF systems.

\subsection{Interaction region layout and magnet designs}

The design of the interaction region of the LHeC (see Fig. 3 for a schematic layout) is particularly challenging as it has to comply with the boundary conditions imposed by the lattice design and optics of the electron and proton beams, the geometry of the LHC tunnel and of the experimental cavern. A complex geometry has to be conceived to allow the collision of the electron beam with the counter clock-wise LHC proton beam (Beam 2 - indicated in red in Fig. 3) while leaving the "spectator" clock-wise proton beam (Beam 1 - indicated in blue in Fig. 3) unperturbed to guarantee operation at the same time of the other LHC proton-proton experiments. This requires the design of superconducting final focus quadrupole magnets providing a high gradient for the colliding proton beam and a near-by field free region for the non-colliding proton 
and electron beams and to minimize synchrotron radiation on the superconducting coils and background to the detector. Possible designs of the triplet quadrupoles have been proposed [1], some of them ("sweet spot magnets") are based on concepts developed for eRHIC [8].

Two dipole low-field $(0.3 \mathrm{~T})$ magnets of opposite polarity, one at each side of the IP and integrated in the detector will provide sufficient separation between the electron and proton beams at the final focus magnets while keeping the synchrotron radiation power and critical energy at acceptable levels.

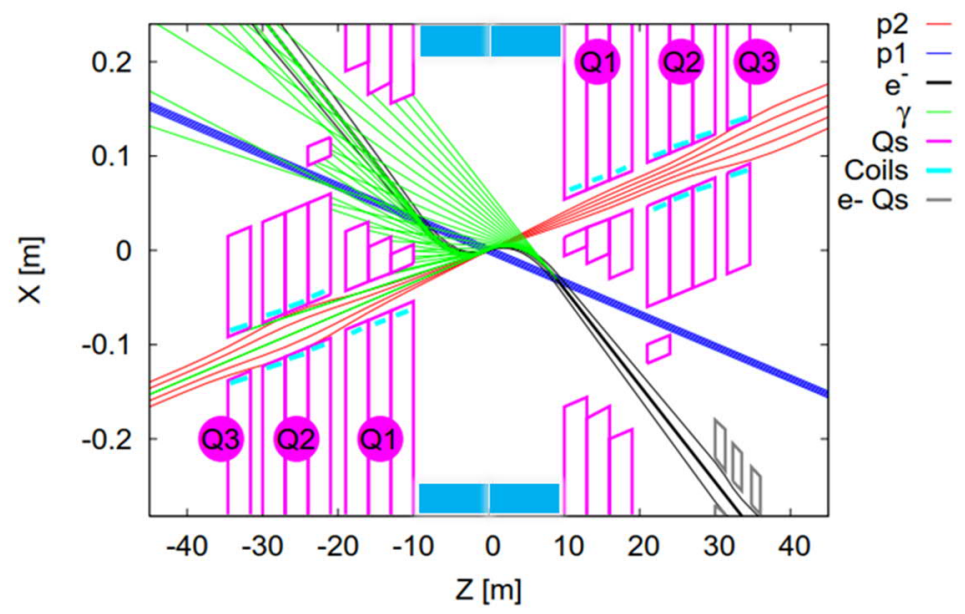

Fig. 3: Schematic layout of the Interaction Region. The low-field separation dipoles embedded in the detector are indicated in light blue.

The design of the interaction regions for $\mathrm{LHeC}$ and $\mathrm{FCC}$-eh is challenging for the minimization of synchrotron radiation, in particular for $\mathrm{LHeC}$. The recent selection of the straight section L for the electron hadron collision IR in FCC makes the design for FCC-eh interaction region more complex taking into account that this straight section accommodates also the injection of the counter-clockwise beam [9].

\subsection{ERL RF System}

The main RF system of the ERL electron accelerator must be operated at a frequency which is a multiple of the frequency corresponding to the LHC bunch spacing $(40.1 \mathrm{MHz})$ in order to guarantee collisions between the electron and proton bunches. In addition it must be taken into account that the 6-passes ERL concept implies that the number of the circulating bunches is six times the number of bunches colliding at the IP and therefore the RF frequency must be at least 6 times higher than the one above mentioned. Other considerations to be taken into account in the choice of the RF frequency are:

- cost, dynamic heat losses, resistance and high-Q $\mathrm{Q}_{0}$ pointing to frequencies between 700 and $800 \mathrm{MHz}[10,11]$;

- beam stability in the presence of Higher Order RF Modes and beam-beam interactions favouring lower frequencies [1].

Three possible SC cavity designs have been considered: Superconducting Proton Linac (704 MHz) [12], ILC (1300 MHz) [13] and FCC/HL-LHC (802 MHz) [3]. $802 \mathrm{MHz}$ has been selected as the $\mathrm{LHeC}$ baseline based on the above considerations and taking into account the synergy with the developments ongoing for HL-LHC/FCC. 
A 5-cell cavity design minimizing High Order Modes (HOM) scaled from a SPL/BNL design has been proposed [14] and a prototype has been built and successfully tested at JLAB as part of a CERN/JLAB collaboration [15]. $\mathrm{Q}_{0}$ values of $3 \times 10^{10}$ have been achieved for RF accelerating fields of up to $27 \mathrm{MV} / \mathrm{m}$, well beyond the requirements of $\mathrm{Q}_{0}=2 \times 10^{10}$ at $18 \mathrm{MV} / \mathrm{m}$ for the LHeC ERL.

\section{ERL electron accelerator demonstrator (PERLE)}

The $\mathrm{LHeC}$ design relies on an efficient operation of the ERL in multi-pass recirculation and energy recovery at unprecedented high current and energy corresponding to a virtual beam power of approximately $1 \mathrm{GW}$. A demonstrator accelerator has been proposed (PERLE: Powerful Energy Recovery Linac for Experiments) with the aim of validating the key LHeC design choices and building-up the expertise in the design and operation of such an accelerator with respect to field quality of the arc magnets, cavity alignment, efficiency of the energy recovery, low-level RF requirements, beam halo and loss minimization, beam stability and general beam diagnostics.

A Conceptual Design Report of the PERLE facility [16] has been published and the main parameters of the accelerator are listed in Table 3 [17].

\begin{tabular}{|l|c|}
\hline Injection energy $[\mathrm{MeV}]$ & 7 \\
\hline Electron beam energy $[\mathrm{MeV}]$ & 500 \\
\hline Normalised Emittance $\gamma \varepsilon_{\mathrm{x}, \mathrm{y}}[\mu \mathrm{m}]$ & 6 \\
\hline Average beam current $[\mathrm{mA}]$ & 20 \\
\hline Bunch charge $[\mathrm{pC}]$ & 500 \\
\hline Bunch length $[\mathrm{mm}]$ & 3 \\
\hline Bunch spacing $[\mathrm{ns}]$ & 25 \\
\hline RF frequency $[\mathrm{MHz}]$ & 801.58 \\
\hline Duty factor & $\mathrm{CW}$ \\
\hline
\end{tabular}

Table 3: Main parameters of the PERLE accelerator

PERLE would be the first superconducting multi-turn ERL accelerator operating with a virtual beam power close to $10 \mathrm{MW}$ demonstrating the ERL concept for $\mathrm{LHeC}$, HE-LHeC, FCCeh and would provide in addition a superconducting RF beam-based development facility and a source of low energy electrons and high energy (few tens of $\mathrm{MeV}$ ) photons for low energy nuclear particle and astro-particle physics. CBETA at Cornell University [18], a 4-pass SRF ERL, should start operation in 2019 with the goal of achieving up to $5 \mathrm{MW}$ beam power but it uses $1.3 \mathrm{GHz}$ SC cavities and FFAG arcs differently from PERLE.

The proposed site for PERLE is the Laboratoire de l'Accelerateur Lineaire (LAL) in Orsay, near Paris, in France and a Collaboration [19-21] is being established with the aim of producing a Technical Design Report and start the construction for demonstrating the LHeC ERL concept in time for operation of the $\mathrm{LHeC}$ during the High Luminosity operation phase of the LHC. 


\section{Acknowledgements}

We have reported here a small fraction of the work performed by the members of the LHeC and FCC-eh Study Groups and the PERLE Collaboration. We would like to thank N. Armesto Perez, A. Bogacz, R. Calaga, D. Douglas, E. Jensen, J. Jowett, W. Kaabi, F. Markhauser, R. Martin, B. Parker, R. Rimmer, D. Schulte, J. Stanyard, M. Stewart, A. Stocchi, C. Tennant, R. Tomas, A. Valloni and F. Zimmermann for providing us with the material for this presentation and paper.

\section{References}

[1] J.L. Abelleira-Fernandez, et al., A Large Electron Hadron Collider at CERN, J. Phys. G: Nucl. Part. Phys. 39 (2012) 075001

[2] O. Brüning, J. Jowett, M. Klein, D. Pellegrini, D. Schulte, F. Zimmermann, Future Circular Collider Study FCC-he Baseline Parameters, CERN-ACC-2017-0019.

[3] G. Apollinari, I. Béjar Alonso, O. Brüning, P. Fessia, M. Lamont, L. Rossi, L. Tavian Eds., HighLuminosity Large Hadron Collider (HL-LHC), Technical Design Report V.0.1, CERN-2017-007-M

[4] F. Zimmermann, O. Bruning, M. Klein, The LHeC as a Higgs Boson Factory, Proceedings of the $4^{\text {th }}$ International Particle Accelerator Conference, Shanghai, China, 12 - 17 May 2013, Z. Dai, C. PetitJean-Genaz, V.R.W. Schaa, C. Zhang, Eds., p. 1017-1019.

[5] D. Amorim et al., High Energy LHC Design, Proceedings of IPAC'18, Vancouver Canada, MOPMF064.

[6] M. Benedikt and F. Zimmermann, Towards Future Circular Colliders, Journal Korean Physical Society 69 (2016) 893.

[7] M. Benedikt, F. Zimmermann, FCC: Colliders at the Energy Frontier, Proceedings of IPAC'18, Vancouver Canada, THYGBD1.

[8] B. Parker, Sweet Spot Designs for Interaction Region Septum Magnets, Proceedings of the 7th International Particle Accelerator Conference, Busan, Korea, 8 - 13 May 2016, C. Petit-Jean-Genaz, D. E. Kim, K. S. Kim, I. S. Ko, K. R. Kim, V.R.W Schaa Eds., p. 1196-1198.

[9] J. Osborne, FCC-eh Civil Engineering Developments, presented at the FCC Week 2018, Amsterdam, 9-13 April 2018 , https:/indico.cern.ch/event/656491/contributions/2947249/attachments/1630258/2600666/FCCeh_Civil_Engineering_Developments_003.pptx

[10] O. Brüning, Accelerator Design, presented at the LHeC Workshop, 25-26 June 2015, Chavannes-deBogis, Switzerland, https://indico.cern.ch/event/356714/contributions/844912/attachments/709271/973673/2015 Works hop_plenary accelerator introduction-V1.pptx

[11] F. Marhauser, Frequency Choice for PERLE and Coupler Simulations, presented at PERLE @ Orsay Workshop, 23-24 February 2017, LAL, Orsay, France, https://indico.lal.in2p3.fr/event/3428/contributions/8937/attachments/8245/9702/2017-0223 Frequency Choice.pdf

[12] F. Gerigk Ed., Conceptual Design of the Low-Power and High-Power SPL - A Superconducting HLinac at CERN, CERN-2014-007. 
[13] J. Brau, Y. Okada, N. Walker, A. Djouadi, J. Lykken, K. Monig, M. Oreglia, S. Yamashita, N. Phinney, N. Toge, T. Behnke, C. Damerell, J. Jaros, A Miyamoto, Eds., International Linear Collider reference design report : ILC Global Design Effort and World Wide Study, CERN-2007-006.

[14] R. Calaga, ERL Facility Cavities, presented at the LHeC Workshop, 25-26 June 2015, Chavannes-deBogis,

Switzerland, https://indico.cern.ch/event/356714/contributions/844905/attachments/709264/973661/LHeCWorks hop_Cavity_Calaga.pdf .

[15] F. Marhauser et al., $802 \mathrm{MHz}$ ERL Cavity Design and Development, Proceedings of IPAC'18, Vancouver Canada, THPAL146.

[16] D. Angal-Kalinin et al., PERLE. Powerful Energy Recovery Linac for Experiments. Conceptual design report, J. Phys. G: Nucl. Part. Phys. 45 (2018) 065003.

[17] W. Kaabi, PERLE Facility: Status and Further Steps, presented at the FCC Week 2018, 9-13 April 2018 , Amsterdam, https://indico.cern.ch/event/656491/contributions/2947251/attachments/1631702/2603366/FCC_we ek PERLE facility status Next steps_W-Kaabi.pptx .

[18] G. H. Hofstaetter et al, CBETA Design Report, Cornell-BNL ERL Test Accelerator, arXiv:1706.04245.

[19] PERLE @ Orsay Workshop, 23-24 February 2017, LAL, Orsay, France, https://indico.lal.in2p3.fr/event/3428/timetable/\#20170223.detailed

[20] First PERLE collaboration meeting, 15-16 January 2018, Daresbury, UK, https://indico.cern.ch/event/690826/

[21] Electrons for the LHC - LHeC/FCCeh and PERLE Workshop, 27-29 June 2018, LAL, Orsay, France, https://indico.cern.ch/event/698368/timetable/ 